\title{
TWO-FORMS ON FOUR-MANIFOLDS
}

\author{
WALTER SEAMAN
}

\begin{abstract}
We study the eigenvalues of the Weitzenböck operator on a positively
\end{abstract} curved four-manifold, and give applications to its geometry and topology.

The purpose of this paper is to prove the following results (see §2).

THEOREM 2. Let $M$ be a Riemannian four-manifold. Suppose there is a point $p \in M$ where all sectional curvatures are positive. Then $M$ admits at most one parallel two-form (up to constant multiples).

The standard metrics on $S^{4}, \mathbf{C} P^{2}$, and the (flat) four-torus $T^{4}$, which have 0,1 , and 6 parallel two-forms, show that the hypothesis cannot be weakened to "nonnegative" and the conclusion is the best possible, at least within the framework of parallel forms. Also, this result fails if we replace "sectional curvature" with "Ricci curvature" as $S^{2} \times S^{2}$ shows (we thank Alfred Gray for pointing this out to us).

THEOREM 3. Let $M$ be a compact positively curved four-manifold without boundary. If $M$ admits a parallel two-form $X$, then the only harmonic two-forms on $M$ are of the form $c X, c \in \mathbf{R}$.

COROLlary. A compact positively curved four-manifold $M$ without boundary which has a parallel two-form $X$ is necessarily a topological $\mathbf{C} P^{2}$.

Proof of the Corollary. Assume first $M$ is orientable. By Theorem 2, the only parallel two-forms on $M$ are $c \cdot X, c \in \mathbf{R}$. By Theorem $3, c \cdot X$ is the only harmonic two-form on $M$. Hence $H^{2}(M ; \mathbf{R})=\mathbf{R}$ by Hodge's theorem. Since any positively curved orientable even dimensional compact manifold is simply connected, we obtain $H_{2}(M ; \mathbf{Z})=\mathbf{Z}$. Hence the intersection form of $M$ is $\{+1\}$ or $\{-1\}$. By Freedman's work [2], $M$, as it has the intersection form class of $\mathbf{C} P^{2}$ or $-\mathbf{C} P^{2}$, and as $M$ was assumed smooth, must be a topological $\mathbf{C} P^{2}$.

If $M$ is not orientable then its two-fold cover is $\mathbf{C} P^{2}$, as above. But $\chi\left(C P^{2}\right)=3$ $=2 \chi(M)$, a contradiction. Q.E.D.

Both Theorems 2 and 3 follow from Theorem 1, a linear algebraic result which states that at any point of positive curvature, if the Weitzenböck operator has kernel, then it has kernel dimension exactly one, and is positive definite on the orthogonal complement.

Received by the editors January 16, 1986 and, in revised form, July 26, 1986. 1980 Mathematics Subject Classification (1985 Revision). Primary 53C20.

(C1987 American Mathematical Society $0002-9939 / 87 \$ 1.00+\$ .25$ per page 
Using Theorem 3 (and not the full strength of the corollary) we can give a new proof of a result originally appearing in [1]:

THEOREM. A compact, positively curved, real four-dimensional Kähler manifold is biholomorphic to $\mathbf{C} P^{2}$.

Proof. Theorem 3 shows that an oriented, compact positively curved fourmanifold $M$, which has a parallel two-form, satisfies $b_{2}(M, \mathbf{R})=1$. Hence $H_{2}(M ; \mathbf{Z})$ $=\mathbf{Z}$ and, since the intersection form is \pm 1 , a result of Whitehead (cf. [4]) implies that $M$ is homotopy equivalent to $C P^{2}$. According to Yau [6], a Kähler homotopy $\mathrm{C} P^{2}$ is biholomorphic to $\mathrm{CP}_{2}$. Q.E.D.

We should also mention that an oriented four-manifold with a parallel two-form $X$ is automatically Kähler (an appropriate constant multiple of $X \pm * X$ will serve as the Kähler form). However, we never use the fact that $M$, if oriented, is complex, since our ultimate goal is to try to understand the number of harmonic two-forms, where no complex structure is available a priori.

We would like to express our gratitude to Dick Randell and Dennis Roseman for many helpful topological discussions.

1. Preliminaries. Let $M$ be an $n$-dimensional Riemannian manifold with curvature tensor $R$, and let $\mu$ be an $r$-form on $M$. Define an $r$-tensor $\mathscr{R}_{r} \mu$ at each $p \in M$ as follows:

$$
\mathscr{R}_{r} \mu\left(v_{1}, \ldots, v_{r}\right)(p)=\sum_{i=1}^{n} \sum_{j=1}^{r}\left(R\left(e_{i}, v_{j}\right) \mu\right)\left(v_{1}, \ldots, v_{j-1}, e_{i}, v_{j+1}, \ldots, v_{r}\right)(p),
$$

where $v_{j} \in T_{p} M, j=1, \ldots, n$, and $\left\{e_{i}\right\}$ forms an orthonormal basis for $T_{p} M$. Then we have the well-known Weitzenböck formula [5, p. 159]

$$
\Delta \mu=-\operatorname{div} \nabla \mu+\mathscr{R}_{k} \mu .
$$

In particular, if $\mu$ is parallel $(\nabla \mu=0)$, then it is also harmonic $(\Delta \mu=0)$ and (2) reduces to $\mathscr{R}_{k} \mu=0$. We now examine (1) in case $r=2$. Using the fact that $R \circ \mu=-\mu \circ R, R$ acts as a derivation on $\Lambda^{r}\left(T_{p} M\right)$, and the Bianchi identity, (1) yields that $\mathscr{R}_{2}$ acts on $\Lambda^{2}\left(T_{p} M\right)$ as follows:

$$
\begin{aligned}
\left\langle\mathscr{R}_{2}\left(X_{1} \wedge X_{2}\right), v_{1} \wedge v_{2}\right\rangle= & \operatorname{Ric}\left(v_{1}, X_{1}\right)\left\langle v_{2}, X_{2}\right\rangle+\operatorname{Ric}\left(v_{2}, X_{2}\right)\left\langle v_{1}, X_{1}\right\rangle \\
& -\operatorname{Ric}\left(v_{1}, X_{2}\right)\left\langle v_{2}, X_{1}\right\rangle-\operatorname{Ric}\left(v_{2}, X_{1}\right)\left\langle v_{1}, X_{2}\right\rangle \\
& -2\left\langle R\left(X_{1}, X_{2}\right) v_{2}, v_{1}\right\rangle,
\end{aligned}
$$

where $X_{i}, v_{j} \in T_{p} M, i, j=1,2$, and Ric $=$ Ricci curvature. Note that $\mathscr{R}_{2}$ is symmetric. Our change from two-forms (elements of $\Lambda^{2}\left(T_{p} M^{*}\right)$ ) to two-vectors (elements of $\left.\Lambda^{2} T_{p} M\right)$ is just a matter of notational convenience. We have used the metric dual, $X=\mu^{\#} \in \Lambda^{2} T_{p} M$, with $X=\sum X_{i} \wedge X_{j}, X_{i}, X_{j} \in T_{p} M$, in the "simple" case $X=X_{1} \wedge X_{2}$. Throughout most of the rest of the paper we shall simply identify two forms with two vectors.

\section{Theorems and proofs.}

TheOREM 1. Suppose $p \in M^{4}$ such that $K(p)>0$, where $K(p)$ is the sectional curvature function. If $\mathscr{R}_{2} X=0, X \neq 0$, and $\mathscr{R}_{2} Y=r Y$, with $0 \neq Y \perp X$, then $r>0$, where $X, Y \in \Lambda^{2} T_{p} M$. Hence, if $0 \neq Z \perp X$, then $\left\langle\mathscr{R}_{2} Z, Z\right\rangle>0$. 
Before we prove this linear algebraic fact, let us go on to deduce the main theorems of the paper.

THEOREM 2. Let $M$ be a Riemannian four-manifold with a point where all sectional curvatures are positive. Then $M$ admits at most one parallel two-form.

Proof. If $X$ and $Y$ are nonzero, independent parallel two-forms, then we may assume that they are orthonormal. By (2), $\mathscr{R}_{2} X=\mathscr{R}_{2} Y=0$. This contradicts Theorem 1. Hence $X=f Y$. But as $X$ and $Y$ are parallel, $f=$ constant. Q.E.D.

THEOREM 3. Let $M$ be a compact positively curved four-manifold (without boundary). If $M$ admits one parallel two-form $X$, then the only harmonic two-forms on $M$ are of the form $c \cdot X, c \in \mathbf{R}$.

Proof. Suppose $X$ is a nonzero parallel two-form on $M$, and $Y$ is a harmonic two-form. As $\mathscr{R}_{2} X=0$, (2) can be used to deduce that $\Delta\langle X, Y\rangle=0$, so $\langle X, Y\rangle$ is constant. If at some point $p, c X_{p} \neq Y_{p}$, we may then replace $Y$ by $Y-$ $\langle X, Y\rangle(p) X /|X|^{2}$, which is still harmonic, and assume that $X$ and $Y$ are globally orthogonal. Then Theorem 1 shows that: for all $p \in M$, either $Y_{p}=0$, or $Y_{p} \neq 0$ and then $\left\langle R_{2} Y, Y\right\rangle(p)>0$. The well-known Bochner argument then shows that this is impossible unless $Y \equiv 0$, since we must have $\int_{M}\left\langle R_{2} Y, Y\right\rangle \leqslant 0$. We may therefore assume that $Y=f \cdot X$. But as $\langle X, Y\rangle$ is constant, $f$ is constant. Q.E.D.

Proof of TheORem 1 . We may assume that $1 \geqslant K(p) \geqslant \delta>0$.

Suppose that $X$ and $Y$ are unit eigenvectors for $R_{2}$, where $R_{2} X=0$ and $R_{2} Y=r Y$, and $\langle X, Y\rangle=0$. There is an orthonormal basis $\left\{e_{1}, \ldots, e_{4}\right\}$ of $T_{p} M$ such that $X=\cos \varphi e_{12}+\sin \varphi e_{34}$, where $e_{i j}=e_{i} \wedge e_{j}$ and $\cos \varphi \geqslant \sin \varphi \geqslant 0$. Using (3), we obtain

$$
0=\left\langle R_{2} X, e_{12}\right\rangle=\cos \varphi K^{12}-2 \sin \varphi R_{1243}
$$

where $K^{12}=K_{13}+K_{14}+K_{23}+K_{24}, \quad K_{i j}=$ sectional curvature of the plane spanned by $e_{i}$ and $e_{j}$, and $R_{i j k l}=\left\langle R\left(e_{i}, e_{j}\right) e_{k}, e_{l}\right\rangle$. Similarly, $0=\left\langle R_{2} X, e_{34}\right\rangle=$ $\sin \varphi K^{12}-2 \cos \varphi R_{1243}$. These equations yield $\left(\cos ^{2} \varphi-\sin ^{2} \varphi\right) R_{1243}=0$. This implies $\cos \varphi=\sin \varphi=\sqrt{2} / 2, R_{1234} \neq 0$, for otherwise the first equation yields $0=$ $K^{12} \geqslant 4 \delta>0$. We therefore have

$$
\begin{gathered}
X=(\sqrt{2} / 2)\left(e_{12}+e_{34}\right), \\
0=K^{12}-2 R_{1243} .
\end{gathered}
$$

Suppose now $r \leqslant 0$ in $R_{2} Y=r Y$. Writing $Y=\cos \psi f_{12}+\sin \varphi f_{34}, f_{i}$ orthonormal, and arguing as before, we find

$$
Y=(\sqrt{2} / 2)\left(f_{12}+f_{34}\right) .
$$

We can define a $*$-operator at $p$ by declaring that $\left\{e_{1}, e_{2}, e_{3}, e_{4}\right\}$ is + oriented. Then $* X=X$ and $* Y= \pm Y$ according as $\left\{f_{1}, f_{2}, f_{3}, f_{4}\right\}$ is \pm oriented. Suppose first that $* Y=-Y$, and write $Y$ relative to the $\left(e_{i}\right)$ basis. We obtain

$$
Y=a\left(e_{12}-e_{34}\right)+b\left(e_{13}+e_{24}\right)+c\left(e_{14}-e_{23}\right),
$$


with $a^{2}+b^{2}+c^{2}=\frac{1}{2}$. A computation then shows that $X+Y$ is simple: $(X+Y)$ $\wedge(X+Y)=0$. Hence for some orthonormal basis $\left\{h_{i}\right\}, X+Y=\alpha h_{12}, \alpha \neq 0$. But then we have $\left\langle R_{2}(X+Y), X+Y\right\rangle=0+r \leqslant 0$, while $\left\langle R_{2}(X+Y), X+Y\right\rangle$ $=\alpha^{2}\left\langle R_{2} h_{12}, h_{12}\right\rangle=$ (by (3)) $\alpha^{2} K^{12}$ (relative to $\left.h_{i}\right) \geqslant \alpha^{2} 4 \delta>0$. Therefore, with $r \leqslant 0,(7), * Y=-Y$ is impossible. Suppose now that $* Y=Y$. Using $\langle X, Y\rangle=0$, we find that

$$
Y=a\left(e_{13}-e_{24}\right)+b\left(e_{14}+e_{23}\right) .
$$

Here $a^{2}+b^{2}=\frac{1}{2}$. Write $a=\sqrt{2} / 2 \cos \vartheta$ and $b=\sqrt{2} / 2 \sin \vartheta$. Let $F_{1}=\cos \vartheta e_{1}+$ $\sin \vartheta e_{2}, F_{2}=\sin \vartheta e_{1}-\cos \vartheta e_{2}$, and $F_{i}=e_{i}, i=3,4$. Then a computation shows

$$
\begin{gathered}
X=(\sqrt{2} / 2)\left[-F_{12}+F_{34}\right], \\
Y=(\sqrt{2} / 2)\left[F_{13}+F_{24}\right] .
\end{gathered}
$$

Now use (3) to expand out $0=\left\langle R_{2} X, F_{12}\right\rangle,(\sqrt{2} / 2) r=\left\langle R_{2} Y, F_{12}\right\rangle$. We obtain

$$
\begin{aligned}
& 0=K^{12}+2 R_{1243}, \\
& r=K^{13}-2 R_{4213},
\end{aligned}
$$

where $K^{13}=K_{12}+K_{14}+K_{23}+K_{34}$, and all these terms are taken relative to the orthonormal $\left\{F_{i}\right\}$ basis. Adding (11) to (12), using the Bianchi identity, and the scalar curvature $\rho=2 \Sigma_{i<j} K_{i j}$, we obtain

$$
r=\frac{1}{2} \rho+K_{14}+K_{23}+2 R_{1423} .
$$

Now the scalar curvature may be computed relative to any orthonormal basis. In particular, letting $K_{i+j, a+b}=$ the sectional curvature of the plane spanned by $\left(F_{i}+F_{j}\right) / \sqrt{2}$ and $\left(F_{a}+F_{b}\right) / \sqrt{2}$, we have

$$
\frac{1}{4} \rho=\frac{1}{2}\left[K_{4+3,1-2}+K_{4+3,1+2}+K_{4-3,1-2}+K_{4-3,1+2}+K_{34}+K_{12}\right]
$$

(scalar curvature computed relative to $\left.\left(F_{4} \pm F_{3}\right) / \sqrt{2},\left(F_{1} \pm F_{2}\right) / \sqrt{2}\right)$,

(15) $\frac{1}{4} \rho=\frac{1}{2}\left[K_{4+2,1-3}-K_{4+2,1+3}+K_{4-2,1-3}+K_{4-2,1+3}+K_{13}+K_{24}\right]$

(scalar curvature computed relative to $\left.\left(F_{4} \pm F_{2}\right) / \sqrt{2},\left(F_{1} \pm F_{3}\right) / \sqrt{2}\right)$. Finally (cf. [3]),

$$
\begin{aligned}
& R_{1423}=\frac{1}{6}\left[K_{1+3,2+4}+K_{1-3,4-2}+K_{4-3,1+2}+K_{4+3,1-2}\right. \\
&-\left.K_{1-3,4+2}-K_{1+3,4-2}-K_{4+3,1+2}-K_{4-3,1-2}\right] .
\end{aligned}
$$

Using (14) plus (15) as the $\frac{1}{2} \rho$ in (13), and using (16) for $R_{1423}$, (13) becomes

$$
\begin{aligned}
0 \geqslant & r=\frac{1}{6}\left[K_{1-3,4+2}+K_{1+3,4-2}+K_{4+3,1+2}+K_{4-3,1-2}\right] \\
& +\frac{5}{6}\left[K_{4+3,1-2}+K_{4-3,1+2}+K_{1-3,4-2}+K_{1+3,4+2}\right] \\
& +\frac{1}{2}\left[K_{34}+K_{12}+K_{13}+K_{24}\right]+K_{14}+K_{23} \\
\geqslant & 8 \delta>0 .
\end{aligned}
$$

This final contradiction shows that $r>0$.

\section{REFERENCES}

1. T. Frankel, Manifolds with positive curvature, Pacific J. Math. 11 (1961), 165-174.

2. M. Freedman, The topology of four dimensional manifolds, J. Differential Geom. 17 (1982), 357-454.

3. H. Karcher, A short proof of Berger's curvature tensor estimates, Proc. Amer. Math. Soc. 26 (1970), $642-644$. 
4. J. Milnor and D. Husemoller, Symmetric bilinear forms, Ergeb. Math. Grenzgeb., Bd. 73, Springer, 1973.

5. W. Poor, Differential geometric structures, McGraw-Hill, 1981.

6. S. T. Yau, On Calabi's conjecture and some new results in algebraic geometry, Proc. Nat. Acad. Sci. U.S.A. 74 (1977), 1748-1799.

Department of Mathematics, University of Iowa, Iowa City, Iowa 52242 